\title{
A Superconducting Transformer System for High Current Cable Testing
}

A. Godeke, ${ }^{1, a)}$ D. R. Dietderich, ${ }^{1}$ J. M. Joseph, ${ }^{1}$ J. Lizarazo, ${ }^{1}$ S. O. Prestemon, ${ }^{1}$ G. Miller, ${ }^{2}$ and H. W. Weijers ${ }^{2}$

1) Lawrence Berkeley National Laboratory, One Cyclotron Road, Berkeley, CA 94720

2) National High Magnetic Field Laboratory, 1800 E. Paul Dirac Drive, Tallahassee, FL 32310

(Dated: 9 July 2010)

This article describes the development of a Direct-Current (DC) superconducting transformer system for the high current test of superconducting cables. The transformer consists of a core-free 10,464 turn primary solenoid which is enclosed by a 6.5 turn secondary. The transformer is designed to deliver a $50 \mathrm{kA}$ DC secondary current at a DC primary current of about $50 \mathrm{~A}$. The secondary current is measured inductively using two toroidal-wound Rogowski coils. The Rogowski coil signal is digitally integrated, resulting in a voltage signal that is proportional to the secondary current. This voltage signal is used to control the secondary current using a feedback loop which automatically compensates for resistive losses in the splices to the superconducting cable samples that are connected to the secondary. The system has been commissioned up to $28 \mathrm{kA}$ secondary current. The reproducibility in the secondary current measurement is better than $0.05 \%$ for the relevant current range up to $25 \mathrm{kA}$. The drift in the secondary current, which results from drift in the digital integrator, is estimated to be below $0.5 \mathrm{~A} / \mathrm{min}$. The system's performance is further demonstrated through a voltage-current measurement on a superconducting cable sample at $11 \mathrm{~T}$ background magnetic field. The superconducting transformer system enables fast, high resolution, economic, and safe tests of the critical current of superconducting cable samples.

PACS numbers: $74.25 . \mathrm{Sv}, 84.37 .+\mathrm{q}, 84.71 . \mathrm{Fk}$

Keywords: superconducting, transformer, high current, cable test

\section{INTRODUCTION}

Critical current $\left(I_{\mathrm{c}}\right)$ tests on superconducting cables for High Energy Physics and Fusion magnets as a function of magnetic field $(H)$ and/or transverse pressure, commonly require currents on the order of tens of kilo-ampères in combination with sub-microvolt resolution voltage measurements and large transverse applied magnetic fields and mechanical loads. A common method to supply large sample currents is through roomtemperature switched current supplies, which are relatively noisy, require large current feedthroughs, and a reliable quench protection system. The high noise of the power supply renders accurate voltage measurement difficult, the large feedthroughs result in high thermal loads on the cryogenics, and faults in the quench protection system can result in sample failure. These disadvantages can be mitigated by using a DC superconducting transformer system as the sample current supply.

DC superconducting transformers are constructed from a primary and secondary coil and a secondary current measurement and feedback system that control the secondary current. The secondary forms a closed nearperfect superconducting loop and therefore acts as an integrator. However, inevitable splice resistances in the secondary closed loop cause the secondary current to decay for a constant current in the primary coil. These resistive losses in the secondary have to be compensated for

a) Electronic mail: agodeke@lbl.gov by a slowly increasing primary current in order to retain a constant secondary current. An accurate compensation requires an accurate measurement of the secondary current, and this forms the heart of the system.

A resistive measurement of the secondary current is impractical due to the large secondary current and the resulting large losses in the system. The secondary current is therefore usually inductively measured by passing it through the aperture of a toroidal-wound Rogowski coil $^{1}$, which generates a voltage signal that is proportional to the time derivative of the secondary current:

$$
U_{\mathrm{r}}=-M_{\mathrm{sr}} \frac{\mathrm{d} I_{\mathrm{s}}}{\mathrm{d} t}
$$

in which the mutual inductance between the secondary and the Rogowski coil $\left(M_{\mathrm{sr}}\right)$ is the proportionality constant. The Rogowski voltage needs to be integrated to generate a voltage that is proportional with the secondary current.

Analog integrators rely on capacitors to retain their charge and therefore do not provide sufficient long term stability of the integrated signal. Early systems ${ }^{2,3}$ therefore use a closed, perfectly superconducting loop to perform the integration. In these systems, the Rogowski coil is superconducting and connected to a superconducting sensing coil, which is enclosed by a superconducting shield to prevent time-dependent stray fields from influencing the measurement. The sensing coil generates a magnetic field that is proportional to the secondary current and this field is detected by a Hall sensor. The Hall voltage is used to drive a measurement current that is looped multiple times through the Rogowski coil and compensates for the secondary current signal. In this 
way, a nulling system is created that is independent of the non-linear field dependence of the Hall-probe voltage and the measurement current is now proportional to the secondary current. This method of secondary current sensing has been applied successfully in a multitude of superconducting transformer systems ${ }^{4-6}$, but requires relatively complex cryogenic instrumentation, and is impractical to debug if any errors occur.

Advances in digital electronics have enabled the development of digital integrators with sufficient long-term stability to be suitable for inductive DC current measurement systems. A more recent $50 \mathrm{kA}$ superconducting transformer system for the FRESCA facility at CERN, Geneva, Switzerland, has demonstrated the feasibility of a 12 bit digital integrator to integrate the signal of a normal conducting Rogowski coil ${ }^{7}$. A smaller scale, 100 A system, constructed around a 16 bit PCI board that performs a digital integration of the Rogowski signal, has also been successfully tested ${ }^{8}$.

Here, we describe the development of a DC superconducting transformer system, which is designed to deliver up to $50 \mathrm{kA}$ for cable tests under transverse pressure in the $12 \mathrm{~T}$ split pair magnet facility at the National High Magnetic Field Laboratory, Tallahassee, FL, USA (NHMFL). The system can easily be adapted for use in other facilities. In section II the design of the superconducting transformer system is described, and test measurements are presented in section III. Overall conclusions are presented in section IV.

\section{DESIGN}

\section{A. Design considerations}

Superconducting cables generally carry currents in the range of 10 to $30 \mathrm{kA}$ in the available background magnetic fields of 8 to $12 \mathrm{~T}$. A suitable target current for the transformer with sufficient margin is therefore on the order of $50 \mathrm{kA}$. Such currents can be carried by a single NbTi cable for the secondary. Output currents on the order of $100 \mathrm{~A}$ are common for relatively compact linear current supplies, and such currents can be carried by simple copper current leads, and by superconducting $\mathrm{NbTi}$ wires that are suitable for the primary windings. A suitable transformer ratio is therefore on the order of 1000 .

The superconducting transformer has to be compact with limited height, to limit the required liquid helium level above the magnet system that contains the cable experiment. The system should allow for the use of two Rogowski coils in anti-series to enable compensation for possible parasitic pickup of the stray magnetic field of the transformer, as was done for the FRESCA facility transformer system ${ }^{7}$. Normal conducting Rogowski coils with a room temperature digital integration are desirable to simplify the cold parts of the current metering system. The current meter system has to include a calibration

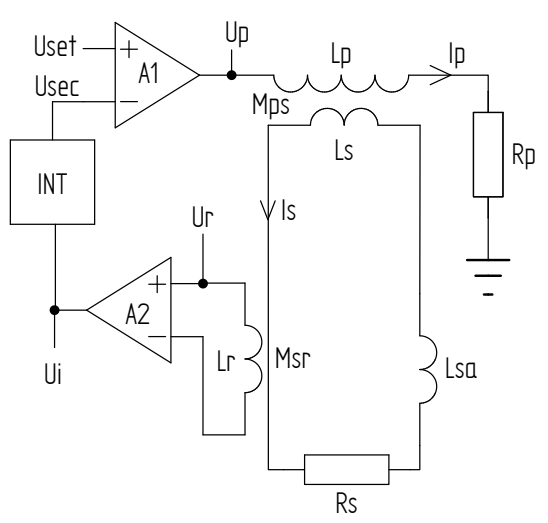

FIG. 1. Simplified schematic of the superconducting transformer and feedback system.

option for the inductive current measurement.

The primary current has to be accurately controlled using a feedback system to allow for automatic compensation of the parasitic losses that occur in the splice resistances in the samples. The following sections describe the implementation of the above general design considerations.

\section{B. Transformer design specifications}

A simplified schematic of a superconducting transformer and feedback system is shown in Figure 1. $A_{1}$ represents a differential voltage amplifier in series with a voltage controlled power supply, generating a control voltage $U_{\mathrm{p}}$ for the primary coil. $A_{2}$ represents a differential voltage amplifier, and INT represents a digital integrator. $L_{\mathrm{p}}$ and $L_{\mathrm{s}}$ represent the inductances of the primary and secondary coils, respectively, $L_{\mathrm{sa}}$ represents the inductance of the sample, and $L_{\mathrm{r}}$ the inductance of the Rogowski coils. $M_{\mathrm{ps}}$ and $M_{\mathrm{sr}}$ represent the mutual inductances between the primary and the secondary coils, and between the secondary cable and the Rogowski coils, respectively. $R_{\mathrm{p}}$ and $R_{\mathrm{s}}$ are the total resistances in the primary and secondary, and $I_{\mathrm{p}}$ and $I_{\mathrm{s}}$ are the currents in the primary and the secondary. $U_{\mathrm{i}}$ is the input voltage of the integrator, and $U_{\mathrm{sec}}$ is a voltage proportional to the secondary current $I_{\mathrm{s}} . U_{\mathrm{sec}}$ is subtracted from $U_{\text {set }}$, an externally generated voltage that is used to control the secondary current. 
The relations that describe the system are (1) and:

Primary

$$
U_{\mathrm{p}}+M_{\mathrm{ps}} \frac{\mathrm{d} I_{\mathrm{s}}}{\mathrm{d} t}=I_{\mathrm{p}} R_{\mathrm{p}}+L_{\mathrm{p}} \frac{\mathrm{d} I_{\mathrm{p}}}{\mathrm{d} t} .
$$

Secondary

$$
M_{\mathrm{ps}} \frac{\mathrm{d} I_{\mathrm{p}}}{\mathrm{d} t}=R_{\mathrm{s}} I_{\mathrm{s}}+\left(L_{\mathrm{s}}+L_{\mathrm{sa}}\right) \frac{\mathrm{d} I_{\mathrm{s}}}{\mathrm{d} t} .
$$

Measurement

$$
\begin{aligned}
U_{\text {sec }} & =\int U_{\mathrm{i}} \mathrm{d} t=A_{2} \int U_{\mathrm{r}} \mathrm{d} t \\
& =-A_{2} M_{\mathrm{sr}} I_{\mathrm{s}} .
\end{aligned}
$$

Feedback

$$
U_{\mathrm{p}}=A_{1}\left(U_{\mathrm{set}}-U_{\mathrm{sec}}\right) .
$$

The amplification factor of the transformer can be found by integrating (3) which gives a current amplification of

$$
\frac{I_{\mathrm{s}}}{I_{\mathrm{p}}} \approx \frac{M_{\mathrm{ps}}}{L_{\mathrm{s}}+L_{\mathrm{sa}}}
$$

when ignoring the (small) resistance in the secondary and alternating current (AC) losses, and when $I_{\mathrm{S}}$ is assumed to be initially zero when $I_{\mathrm{p}}=0$ at the beginning of the current ramp.

It is evident from (6) that, though in principle a single turn secondary is sufficient, it is important to use multiple turns to enhance the amplitude of the mutual and secondary inductances, thereby reducing the sensitivity of the amplification factor on the load inductance.

To illustrate the role of load inductance and secondary turn number, consider the cross-section of the secondary being composed of $n_{2}$ turns. Equation (6) then takes the general form

$$
\frac{I_{\mathrm{s}}}{I_{\mathrm{p}}} \approx \frac{n_{2} M_{\mathrm{ps}}}{n_{2}^{2} L_{\mathrm{s}}+L_{\mathrm{sa}}} .
$$

The resulting amplification as a function of secondary turn number is shown in Figure 2, for various load inductance values. Note that the measured amplification factor is a sensitive diagnostic of small load inductance.

The current specification of the primary power supply follows directly from (6) and the desired headroom to compensate for the $\mathrm{AC}$ and resistive losses in the secondary. The voltage requirement for the primary power supply follows from (2), and is therefore determined by the desired ramprate for the secondary current combined with the resistive voltages in the primary current leads.

A NbTi cable for the secondary was custom made from wire that was developed for the U.S. Superconducting Supercollider Program (SSC), and insulated with S-glass braided sleeve. A Formvar insulated NbTi wire from existing inventory was selected for the primary windings. The secondary cable and primary wire specifications are summarized in Table I.

The primary coil is solenoidal wound on a $80 \mathrm{~mm}$ diameter, $150 \mathrm{~mm}$ high stainless steel holder and the secondary coil is wound on top of the primary, with a $2 \mathrm{~mm}$

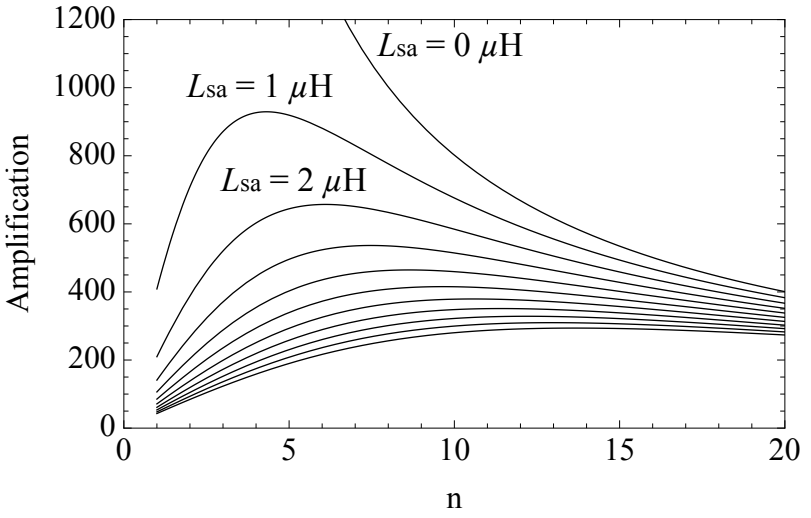

FIG. 2. Amplification factor $I_{\mathrm{s}} / I_{\mathrm{p}}$ as a function of secondary turns $(n)$ for load inductances from 0 to $10 \mu \mathrm{H}$, based on the

\begin{tabular}{|c|c|c|c|c|}
\hline $\begin{array}{c}\text { Cable } \\
\text { dimensions } \\
\mathrm{mm} \times \mathrm{mm}\end{array}$ & $\begin{array}{c}\text { Cable } I_{\mathrm{c}} \\
\text { at } 5 \mathrm{~T} \\
{[\mathrm{kA}]}\end{array}$ & $\begin{array}{l}\text { Number } \\
\text { of strands }\end{array}$ & $\begin{array}{c}\text { Wire } \\
\text { diameter } \\
{[\mathrm{mm}]}\end{array}$ & $\begin{array}{c}\text { Wire } I_{\mathrm{c}} \\
\text { at } 5 \mathrm{~T} \\
{[\mathrm{~A}]}\end{array}$ \\
\hline $1.626 \times 21.267$ & 31.26 & 51 & 0.386 & 110 \\
\hline
\end{tabular}
geometry used in the transformer described here. The actual turn number chosen is $\sim 6.5$, yielding reasonable performance for both low-inductance short samples and higher-inductance test coils.

TABLE I. Secondary cable and primary wire specifications (uninsulated)

insulating spacer in between the primary and secondary turns, and insulated on the outside with S-glass sheet. The primary and secondary windings are optimized for the mutual inductance between the turns. The coil set is clamped inside an Al-alloy support structure and vacuum impregnated with CTD101 epoxy. Table II provides an overview of the calculated transformer specifications.

The design of the transformer is shown in Figure 3, including the Rogowski coils for the current measurement that are described in more detail in section II C. The secondary leads leave the transformer at the top and are folded down and brought together on the side the the transformer to limit the required height of the system. The outer dimensions of the entire transformer, excluding the splices to the sample, are $190 \times 180 \times 220 \mathrm{~mm}^{3}$.

The inductances and mutual inductances of the transformer are calculated from the primary and secondary coil dimensions. The primary resistance $R_{\mathrm{p}}$ is estimated from the voltage drop that is commonly observed in copper welding cable current leads. The secondary resistance $R_{\mathrm{S}}$ is a worst case estimate that is based on past experience that good solder joints between Rutherford cables commonly exhibit resistances of $0.5 \mathrm{n} \Omega$ or lower.

The inductance of a bifilar set of Rutherford cables per unit length is approximately $L \approx \mu_{0} d / w$. Here, $d \approx 2 \mathrm{~mm}$ is the cable center separation and $w \approx 20 \mathrm{~mm}$ is the cable width, leading to $L_{\mathrm{sa}} \approx 1.5 \times 10^{-7} \mathrm{H}$ for 
TABLE II. Transformer system specifications

\begin{tabular}{cccccccccc}
\hline \hline $\begin{array}{c}\text { Primary } \\
\text { \# turns }\end{array}$ & $\begin{array}{c}\text { Secondary } \\
\text { \# turns }\end{array}$ & $\begin{array}{c}L_{\mathrm{p}} \\
{[\mathrm{H}]}\end{array}$ & $\begin{array}{c}R_{\mathrm{p}} \\
{[\mathrm{m} \Omega]}\end{array}$ & $\begin{array}{c}L_{\mathrm{s}} \\
{[\mu \mathrm{H}]}\end{array}$ & $\begin{array}{c}R_{\mathrm{s}} \\
{[\mathrm{n} \Omega]}\end{array}$ & $\begin{array}{c}I_{\mathrm{p}} \\
{[\mathrm{A}]}\end{array}$ & $\begin{array}{c}I_{\mathrm{s}} \\
{[\mathrm{A}]}\end{array}$ & $\begin{array}{c}M_{\mathrm{ps}} \\
{[\mathrm{mH}]}\end{array}$ & $\begin{array}{c}M_{\mathrm{sr}} \\
{[\mu \mathrm{H}]}\end{array}$ \\
\hline 10,464 & 6.5 & 4.5 & $\approx 20$ & 2.27 & $\leq 10$ & 50 & 50,000 & 2.8 & 5.3 \\
\hline \hline
\end{tabular}

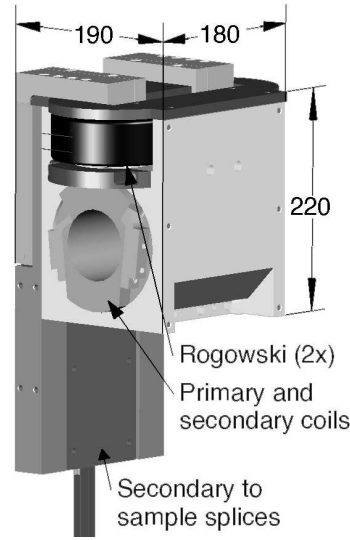

FIG. 3. Artist impression of the superconducting transformer system. Dimensions are in $\mathrm{mm}$.

a $1.2 \mathrm{~m}$ sample length and, using (6), to a current gain of 1157. Rounding this to a gain of 1000 to account for losses yields a design primary current of $50 \mathrm{~A}$ at a target secondary current of $50 \mathrm{kA}$. $U_{\mathrm{p}}$ can be calculated from (2) at a secondary ramprate of $1000 \mathrm{~A} / \mathrm{s}$ (and thus a primary ramprate of $1 \mathrm{~A} / \mathrm{s}$ ) and a primary current of $50 \mathrm{~A}$, yielding $U_{\mathrm{p}}=2.7 \mathrm{~V}$. These order-of-magnitude calculations indicate that a $5 \mathrm{~V}, 50 \mathrm{~A}$ primary power supply (PS) will suffice.

A voltage programmable HP6260B $10 \mathrm{~V}, 100 \mathrm{~A}$ PS is selected for the primary coil. The PS has an adjustable over-voltage protection limit, which triggers a fan cooled, heat-sink mounted Silicon Controlled Rectier (SCR) to short the PS output, thereby effectively limiting the voltage to the SCR P-N junction voltage drop and discharging the stored energy of the transformer over the SCR. Reverse polarity mounted diodes further protect the PS output circuitry against reverse voltages that might occur during a sample and/or transformer quench. The selected PS therefore provides an intrinsic quench detection and protection system for the transformer and sample.

The selected PS can only provide a limited negative voltage (which is required for a stable system feedback at low excitations), does not allow for a bi-polar operation of the transformer, and limits the ramp-down rate of the primary. The added cost of a bi-polar or four quadrant PS does not, for now, justify the benefits.
TABLE III. Approximate Rogowski coil specifications.

\begin{tabular}{lc}
\hline \hline Turns & 1550 \\
Self inductance, $L[\mathrm{mH}]$ & 8.1 \\
Mutual inductance, $M_{\mathrm{sr}}[\mu \mathrm{H}]$ & 5.3 \\
Resistance, $R[\Omega]$ & 210 \\
Self capacitance incl. lead, $C[\mathrm{nF}]$ & 1.3 \\
Resonant frequency [kHz] & 50 \\
Optimum damping resistance, $R_{\mathrm{d}}[\mathrm{k} \Omega]$ & 1 \\
\hline \hline
\end{tabular}

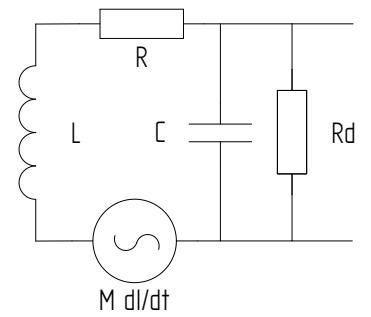

FIG. 4. Electrical equivalent circuit of a Rogowski coil.

\section{Current measurement}

A system based on normal conducting Rogowski coils, combined with a room temperature digital integrator is selected for the secondary current measurement. The Rogowski coils are machine-wound from insulated copper wire by Rocoil Ltd., Harrogate, North Yorkshire, England. The approximate specifications of the Rogowski coils are given in Table III, and an electrical equivalent circuit is shown in Figure 4. The mutual inductance $M_{\mathrm{sr}}$ in Table III is the mutual inductance between the Rogowski coil and a current that is fed through the coil (i.e. the secondary current).

The self inductance of the coils in combination with their parasitic capacitance forms an oscillator with a resonant frequency of about $50 \mathrm{kHz}$. The Rogowski coils are therefore shorted with a damping resistor $R_{\mathrm{d}}=1 \mathrm{k} \Omega$ to reduce high frequency noise. The voltage over the damping resistor is fed into a balanced input of a custom built instrumentation amplifier which is based on a BurrBrown INA128A4 (AMP G2). The amplifier has an adjustable gain G2. A schematic of the entire measurement and control system is shown in Figure 5. The Rogowski signal is low-pass filtered after the instrumentation amplifier using a first order $R C$ filter with a $-3 \mathrm{~dB}$ point at $15 \mathrm{kHz}$, thereby limiting the bandwidth of the current measurement, but preventing high frequency noise from 
entering the digital integrator circuit.

The digital integrator is built using a NI sbRIO9631 board, which contains a real-time $266 \mathrm{MHz}$ processor, a user-reconfigurable field-programmable gate array (FPGA), 32 16-bit analog inputs, and four 16-bit analog outputs. The amplified and conditioned Rogowski signal is fed into a balanced 16 bit analog-to-digital (A/D) convertor and transferred to the FPGA, which is programmed to internally calculate with 64 bit words. The board is programmed using a combination of National Instruments Labview code and custom VHDL code to program the FPGA. The actual integration is performed in real time inside the FPGA using a simple summation and frequency division algorithm. The 64 bit integrated Rogowski signal is then scaled back to 16 bit and converted back to an analog voltage using a 16 bit digitalto-analog $(\mathrm{D} / \mathrm{A})$ convertor, resulting in a voltage $U_{\text {sec }}$ that is proportional with the secondary current $I_{\mathrm{s}}$.

A key factor in digital integrators is to prevent drift in the integrated signal. Drift can occur when a (timevariable) offset is present at the input of the digital integrator. Offset voltages will be present at the output of the instrumentation amplifier and the amplifiers in the A/D convertor. Since manual offset trimming is inaccurate and potentially introduces temperature- and timedependent offset changes, the offset is compensated digitally inside the FPGA. The offset compensation value is captured when the secondary current is zero, which is enabled by switching on the heater $R_{\mathrm{h}}$ in Figure 5 to locally heat the secondary cable above its critical temperature. The offset is measured and averaged for 3 seconds after switch SW2 is momentarily activated. The averaged offset is stored and subtracted from the incoming Rogowski signal before integration. Indicator L1 turns on when the offset has been compensated and the system is ready to measure the secondary current.

The current measurement system is designed to enable the use of two Rogowski coils that are connected in antiseries. An ideal Rogowski coil is insensitive to external magnetic fields, but in practice some sensitivity to external magnetic fields can be expected as a result of winding imperfections. Any parasitic pickup of the stray magnetic field of the primary coil by the Rogowski coil will cause an error in the current measurement, since the primary of the transformer generates a time-varying magnetic field. This potential error can be reduced by mounting two Rogowski coils symmetric but opposite with respect to the primary coil and connecting their output leads in antiseries.

The current measurement is calibrated by feeding a current up to 100 A through a NbTi wire $\left(I_{\text {cal }}\right.$ in Figure 5$)$ that is looped 50 times through the Rogowski coils while the secondary cable is heated above its critical temperature. The calibration current is measured using a Hitec Power Protection bv MACC ${ }^{\text {plus }}$ Zero-flux ${ }^{\text {TM }}$ DC current meter which has a precision of $0.01 \%$. In this way the current measurement is calibrated up to approximately $5 \mathrm{kA}$. The current measurement at higher currents relies on this calibration and linearity of the transfer function of the current measurement (relation 4).

\section{Feedback and current control}

The measured secondary current, represented by voltage $U_{\mathrm{sec}}$, is subtracted from an externally generated control voltage for the secondary current $U_{\text {set }}$ in a second custom built instrumentation amplifier (AMP G1) with an adjustable gain G1 (see Figure 5). This creates a negative feedback loop, in which the error signal (5) is used to control the voltage across the primary coil. In this way, the secondary current will be directly proportional to the control voltage $U_{\text {set }}$. Any drift in the secondary current, which occurs as a result of the losses in the resistive splices to the sample, AC-losses, and losses when the sample is near its critical current, will be automatically compensated for by an increase of the primary current.

The entire current measurement and control system is built inside a single box (the control and readout unit indicated by the dashed line in Figure 5). The digital and analog parts of the system are fed by independent power supplies with floating ground levels. The digital and analog grounds are connected together at a suitable higher signal level point in the control and readout unit and SW1 enables a selectable connection to the mains ground. This allows for two user-selectable grounding options in the system depending on the ground connections in the used PS for the primary coil, thereby preventing undesired ground loops in the system.

\section{E. System bandwidth}

The frequency domain of the system can be studied by performing a Laplace transform on the relations describing the system (2-5), leading to

$$
\begin{aligned}
& \text { Primary } \\
& I_{\mathrm{p}}=\frac{U_{\mathrm{p}}+M_{\mathrm{ps}} I_{\mathrm{s}} s}{R_{\mathrm{p}}+L_{\mathrm{p}} s} . \\
& \text { Secondary } \\
& I_{\mathrm{s}}=\frac{M_{\mathrm{ps}} I_{\mathrm{p}} s}{R_{\mathrm{s}}+\left(L_{\mathrm{s}}+L_{\mathrm{sa}}\right) s} . \\
& \text { Measurement } \\
& U_{\mathrm{sec}}=A_{2} M_{\mathrm{sr}} I_{\mathrm{s}} .
\end{aligned}
$$
quency $\mathrm{i} \omega$. 


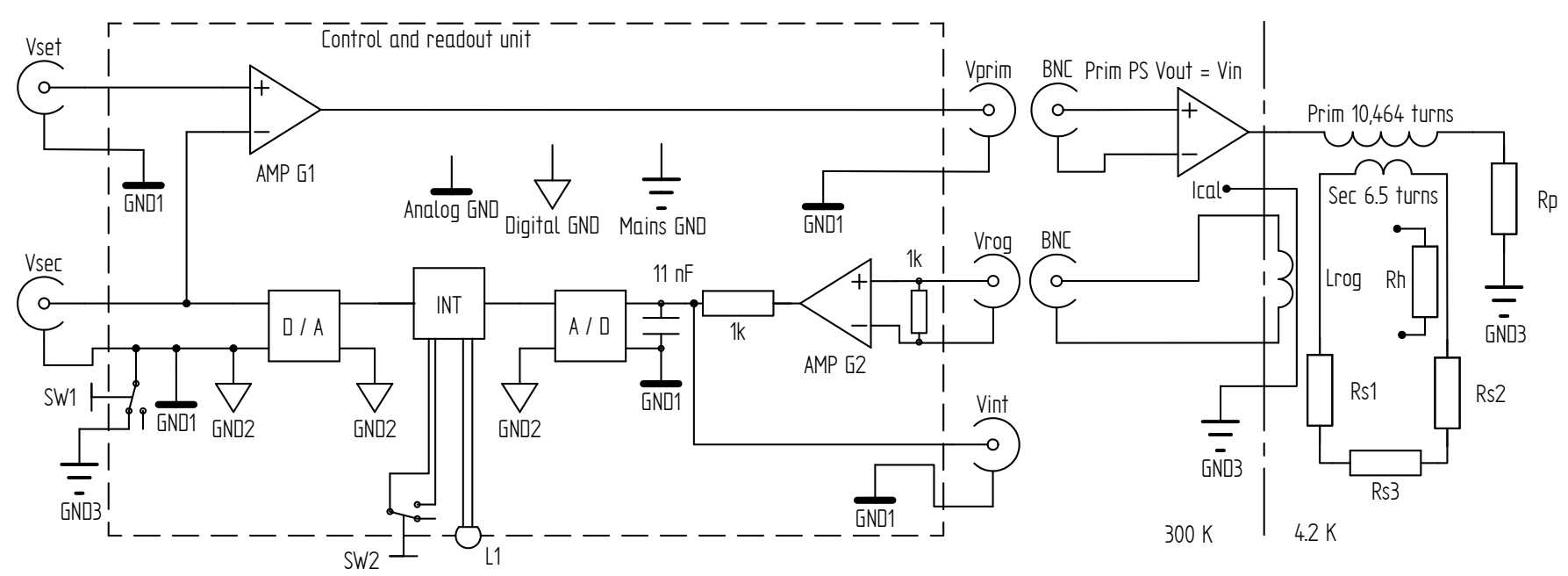

FIG. 5. Electrical schematic of the transformer and control system.

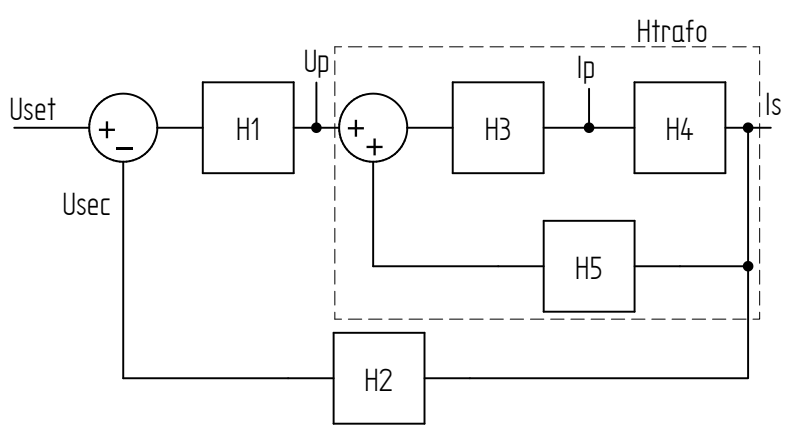

FIG. 6. Block schematic of the transformer and control system.

Relations (8-10) and (5) can be graphically represented in a block diagram (Figure 6) to simplify the determination of the transfer functions, as is common in system analysis. The transfer functions $\mathrm{H}_{i}$ are

$$
\begin{aligned}
\mathrm{H}_{1} & =\mathrm{G} 1 \\
\mathrm{H}_{2} & =\mathrm{G} 2 M_{\mathrm{sr}}, \\
\mathrm{H}_{3} & =\frac{1}{R_{\mathrm{p}}+L_{\mathrm{p}} s}, \\
\mathrm{H}_{4} & =\frac{M_{\mathrm{ps}} s}{R_{\mathrm{s}}+\left(L_{\mathrm{s}}+L_{\mathrm{sa}}\right) s}, \\
\mathrm{H}_{5} & =M_{\mathrm{ps}} s, \\
\mathrm{H}_{\text {trafo }} & =\frac{I_{\mathrm{s}}}{U_{\mathrm{p}}}=\frac{\mathrm{H}_{3} \mathrm{H}_{4}}{1-\mathrm{H}_{3} \mathrm{H}_{4} \mathrm{H}_{5}}, \\
\mathrm{H}_{\text {total }} & =\frac{I_{\mathrm{s}}}{U_{\mathrm{set}}}=\frac{\mathrm{H}_{1} \mathrm{H}_{\text {trafo }}}{1+\mathrm{H}_{1} \mathrm{H}_{\text {trafo }} \mathrm{H}_{2}} .
\end{aligned}
$$

The absolute values of $\mathrm{H}_{\text {trafo }}$ and $\mathrm{H}_{\text {total }}$ now represent their respective amplitude responses as a function of frequency. The arguments of $\mathrm{H}_{\text {trafo }}$ and $\mathrm{H}_{\text {total }}$ now represent their phase responses as a function of frequency. These

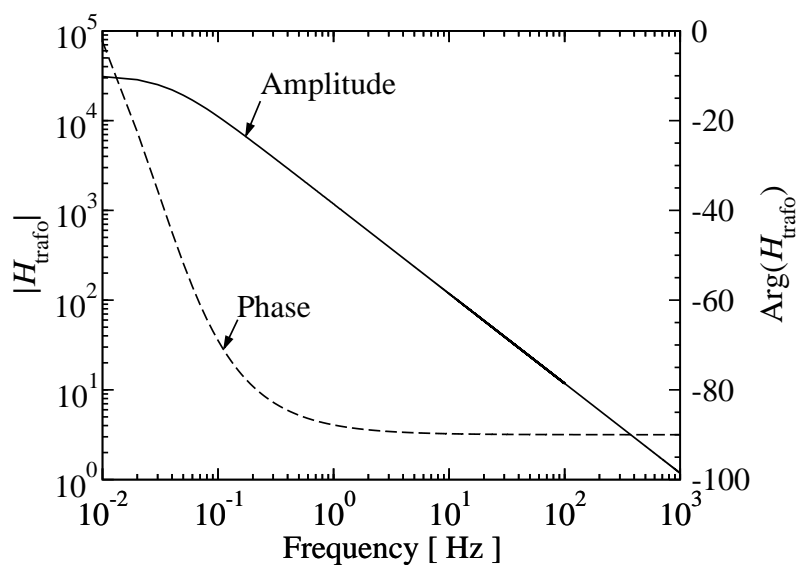

FIG. 7. Amplitude and phase of the transfer function $\mathrm{H}_{\text {trafo }}=$ $I_{\mathrm{s}} / U_{\mathrm{p}}$ of the transformer as a function of frequency.

amplitude and phase responses are plotted in Figures 7 and 8 for $\mathrm{G} 1=10$ and $\mathrm{G} 2=100$.

The frequency response of $\mathrm{H}_{\text {trafo }}$ shows that the transformer behaves almost as a pure DC system. This is beneficial, since any ripple on the primary power supply voltage will not be transferred to the secondary current. It means that the transformer has a rather slow response to changes in the primary voltage. The phase change to -90 degree is due to the switch from primary voltage to secondary current. The response of the secondary current to the primary current is determined by $\mathrm{H}_{4}$ which, though not shown here, has a flat frequency dependence, indicating an immediate change in $I_{\mathrm{s}}$ for a change in $I_{\mathrm{p}}$. The absolute value of this response equals the current amplification factor of 1157 .

The frequency response of the total system $\mathrm{H}_{\text {total }}$, i.e. the response of $I_{\mathrm{s}}$ to a change in the control voltage $U_{\text {set }}$, has a bandwidth of about $10 \mathrm{~Hz}$. The bandwidth can in principle be increased significantly by increasing the 


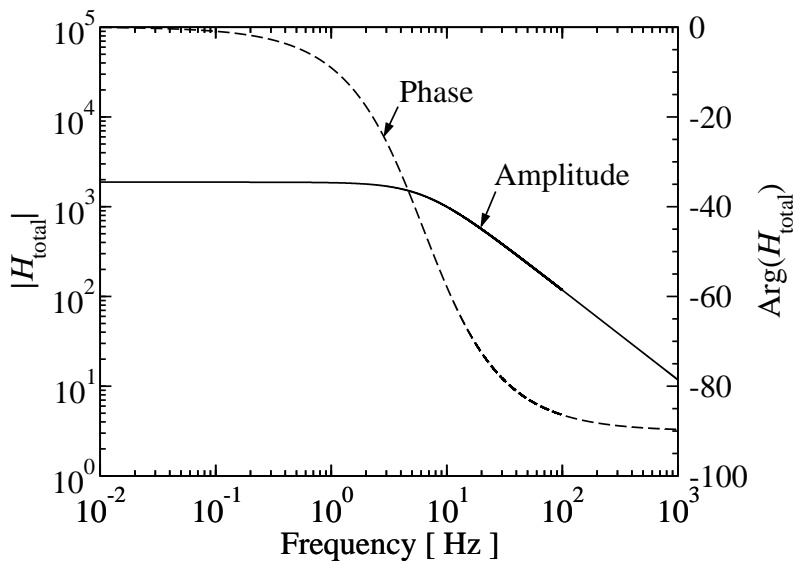

FIG. 8. Amplitude and phase of the transfer function $\mathrm{H}_{\text {total }}=$ $I_{\mathrm{s}} / U_{\text {set }}$ of the total system as a function of frequency.

loop-gain, i.e. by increasing G1 and G2. A gain of 100 in the current measurement amplifier AMP G2 represents, however, a balance between desired amplification of the Rogowski voltage for a desired maximum secondary current measurement, the input range of the $\mathrm{A} / \mathrm{D}$ convertor (which is set at $1 \mathrm{~V}$ full scale), and the output range of the D/A convertor (which is set at $10 \mathrm{~V}$ full scale). An increase in G2 further increases the strength of the feedback and thus reduces the amplitude of $\mathrm{H}_{\text {total }}$.

Though the gain in the feedback amplifier AMP G1 can be increased to increase the bandwidth, it is intentionally kept low to create a softer start and end when changing the secondary current stepwise during the measurement of a voltage-current transition in the sample. Increasing G1 and thus increasing the bandwidth will cause a sharper change in the secondary current when the ramp is started or ended, thereby increasing the possibility of a sample quench during stepwise ramping of the secondary current. The secondary current measurement, however, still has a bandwidth of $15 \mathrm{kHz}$, allowing for the measurement of rapid changes in the secondary current, as occur during a quench of the sample and/or transformer.

\section{PERFORMANCE TESTS}

A number of tests were performed to analyze the performance of the superconducting transformer and control system. First, the current measurement system was calibrated and analyzed for reproducibility. Next, the parasitic coupling between the primary current and the Rogowski coils and the drift in the digital integrator was investigated. This was followed by a determination of the effective current amplification factor. The overall system performance is finally demonstrated by a voltage-current measurement on a $\mathrm{Nb}_{3} \mathrm{Sn}$ Rutherford cable sample.

\section{A. Current calibration}

The current measurement is calibrated by driving a known current through the calibration wire that is looped multiple times through the Rogowski coil, while the secondary cable is heated above its critical temperature. The calibration current is measured using the MACC ${ }^{\text {plus }}$ Zero-flux ${ }^{\mathrm{TM}}$ DC current meter, which provides an output current that is a factor 1000 lower than the current that is to be measured. The accuracy of the ratio between the output current and the unknown current is better than $100 \mathrm{ppm}$. The overall uncertainty in the measurement of the calibration current, which includes the uncertainty in the measurement of the output current of the Zeroflux, is below $0.01 \%$. The calibration current was ramped up with $20 \mathrm{~A} / \mathrm{s}$, which translates to $1000 \mathrm{~A} / \mathrm{s}$ since the wire is looped 50 times through the Rogowski coils. The current was ramped up to around $80 \mathrm{~A}$, or $4 \mathrm{kA}$ effectively, held for a few seconds, and then ramped down again at an effective ramp-rate of $1000 \mathrm{~A} / \mathrm{s}$. The high ramp-rate was selected to increase the signal-to-noise ratio and the hold time was chosen to be short, to ensure that the calibration is not influenced by possible drift in the integrator.

The results of the calibration, for a selected set of G2 gain values and ramp-rates are summarized in Table IV. Other gain settings and ramp-rates indicate comparable results. The calibration is performed for each individual Rogowski coil, as well as for a anti-series connection of both coils. The sensitivity is averaged over four calibration ramps for each Rogowski coil combination and the maximum deviation of the sensitivity compared to the average sensitivity of the four ramps is used to calculate the reproducibility.

The theoretical sensitivity is $\mathrm{G} 2 M_{\mathrm{sr}}$ for a single coil and $2 \times \mathrm{G} 2 M_{\mathrm{sr}-\text { average }}$ for the two coils in anti-series, as follows from (10). This leads to $265 \mathrm{mV} / \mathrm{kA}$ for coil SG-872, $261 \mathrm{mV} / \mathrm{kA}$ for coil SG-864, and to $210 \mathrm{mV} / \mathrm{kA}$ for the anti-series connection of both coils. It is seen from Table IV that the measured sensitivities for the single coils are about $4 \%$ higher than the rated sensitivities and about $2 \%$ higher for the anti-series connected coils, indicating that the difference is most probably a result of inaccuracy in the gain G2.

The reproducibility of the current measurement is of the same order of magnitude as the uncertainty in the calibration current measurement, i.e. around $0.01 \%$ or better, indicating a high accuracy of the current measurement with the Rogowski coils and integrator. The uncertainty in the current measurement can be expected to increase proportionally with the current, yielding an estimated uncertainty around $0.02 \%$ to $0.04 \%$ for currents in the 10 to $20 \mathrm{kA}$ range, respectively.

\section{B. Parasitic coupling of the primary to the Rogowski coils}

The parasitic coupling between the primary current and the Rogowski coils can be measured by increasing 
TABLE IV. Current measurement calibration summary

\begin{tabular}{|c|c|c|c|c|c|}
\hline \multicolumn{6}{|c|}{ Rogowski coil and rated mutual inductance } \\
\hline \multicolumn{2}{|c|}{$\mathrm{SG}-872, M_{\mathrm{sr}}=5.297 \mu \mathrm{H}$} & \multicolumn{2}{|c|}{$\mathrm{SG}-864, M_{\mathrm{sr}}=5.224 \mu \mathrm{H}$} & \multicolumn{2}{|c|}{ SG-872 + SG-864 } \\
\hline Gain G2 & Ramp rate & Gain G2 & Ramp rate & Gain G2 & Ramp rate \\
\hline $50 \times$ & $1000 \mathrm{~A} / \mathrm{s}$ & $50 \times$ & $1000 \mathrm{~A} / \mathrm{s}$ & $20 \times$ & $1000 \mathrm{~A} / \mathrm{s}$ \\
\hline Average sensitivity & Reproducibility & Average sensitivity & Reproducibility & Average sensitivity & Reproducibility \\
\hline$[\mathrm{mV} / \mathrm{kA}]$ & {$[\%]$} & {$[\mathrm{mV} / \mathrm{kA}]$} & {$[\%]$} & {$[\mathrm{mV} / \mathrm{kA}]$} & {$[\%]$} \\
\hline $276.08 \pm 0.02$ & 0.01 & $272.26 \pm 0.01$ & 0.004 & $215.07 \pm 0.02$ & 0.006 \\
\hline
\end{tabular}

the primary current while heating the secondary above its critical temperature. This yields a worst-case number for the parasitic coupling between primary and the Rogowski coils, since the magnetic stray field that is generated by the transformer is maximum when the secondary current is zero. In practice, when the secondary is superconducting and allowed to carry its full transformed current during the measurement of a sample, the stray magnetic field of the transformer is significantly lower, since in this case the secondary is absorbing most of the magnetic energy that is generated by the primary.

To determine the worst-case coupling, the secondary is heated and the primary current is increased by applying a constant voltage to the primary coil. The secondary current is measured using only one Rogowski coil (coil SG-872) so that the parasitic coupling is not reduced by an anti-series connection of the two Rogowski coils. A constant $U_{\mathrm{p}}$ results in a constant $\mathrm{d} I_{\mathrm{p}} / \mathrm{d} t$, which in turn generates a constant voltage $U_{\mathrm{s}}$ on the secondary side of the transformer, and therefore a constant current in the secondary $I_{\mathrm{s}}=U_{\mathrm{s}} / R_{\mathrm{s}}$. Any apparent change in the secondary current that is detected by the Rogowski coils and proportional to the change in the primary current, stems from the parasitic coupling between the primary and the Rogowski coils.

A measurement of $I_{\mathrm{p}}$ and $I_{\mathrm{s}}$ as a function of time $t$ with the secondary heater activated is shown in Figure 9. Here, the voltage over the primary coil is stepwise increased at $t \approx 0,22,57, \ldots$ etcetera seconds. At $t \approx 160 \mathrm{~s}, U_{\mathrm{p}}$ is switched from positive to negative. At the beginning of the primary $\operatorname{ramp} U_{\mathrm{s}}=M_{\mathrm{ps}} \mathrm{d} I_{\mathrm{p}} / \mathrm{d} t=$ $2.8 \times 10^{-3} \times 0.068=0.2 \mathrm{mV}$ and $I_{\mathrm{s}}=48.5 \mathrm{~A}$, yielding $R_{\mathrm{S}} \approx 4 \mu \Omega$. This shows that the normal state resistance of the heated secondary section is about four orders of magnitude larger than the splice resistances, which are typically $\leq 0.5 \mathrm{n} \Omega$ each. Note that the inductive component of the secondary (see eq. 3 ) will decay with a time constant $\left(L_{s}+L_{s a}\right) / R_{s} \approx 0.6 \mathrm{~s}$, significantly shorter than seen in Fig 9, and hence can be neglected in this analysis of field-coupling to the Rogowski coil.

The visible change in the secondary current measurement in Figure 9, which is inversely proportional to the change in the primary current, is due to the parasitic coupling between the primary current and the Rogowski coil SG-872. The average value of the proportionality constant is -2.6 Asec/Aprim. This worst-case value indi-

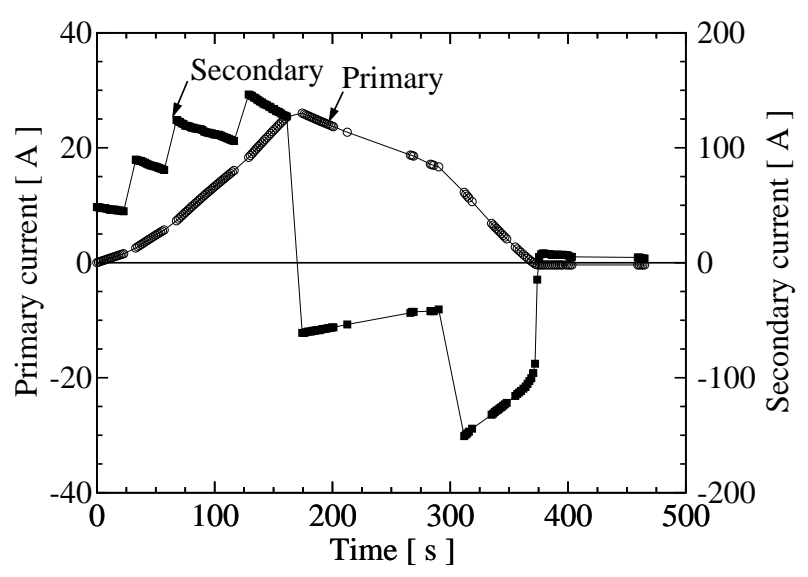

FIG. 9. Secondary current as a function of primary current with the secondary heater switched on.

cates that, for example, at a secondary current of $10 \mathrm{kA}$, and thus a primary current of about $10 \mathrm{~A}, \mathrm{a}-26 \mathrm{~A}$ error occurs in the measurement of the secondary as a result of the parasitic coupling between the primary and the Rogowski coil. This error is negligible, indicating a homogeneously wound Rogowski coil.

During a sample measurement most of this error disappears, as a result of the reduction in the transformer magnetic stray field by the secondary current. The error can in principle be further minimized through the use of both Rogowski coils and connecting them in anti-series, but the measurement above indicates that the error, even in the worst-case scenario, is acceptably small, and therefore that one Rogowski coil is sufficiently accurate.

\section{Drift in the current measurement}

The drift in the current measuring system was determined in two ways. First the drift in the stand-alone electronics was determined, and then the drift during a current measurement was analyzed.

The drift in the measurement and control unit was determined without attaching a Rogowski coil at the input of AMP G2 in Figure 5. The drift was thus measured with the input of AMP G2 shorted with the $1 \mathrm{k} \Omega$ damping resistor. The offset was nulled with an oscilloscope 
connected to the output of the D/A convertor. After 15 hours, no drift was observed within the $1 \mathrm{mV}$ resolution of the oscilloscope. It should be noted that an accurate compensation of the offset depends on how representative the offset over the 3 second nulling period is for the offset in the remainder of the experiment. Any disturbances such as thermal instabilities, or the pickup of spikes and noise during the 3 second period, will render the offset compensation invalid. In practice, when this occurs, a clearly detectable drift is immediately visible at the output of the integrator after the offset determination period.

An example of the integrator drift in the total measuring system, with the Rogowski coil attached, is visible in Figure 9. The secondary current measurement does not completely return to zero when the primary current has returned to zero, which is a result of drift in the secondary current measurement. The drift in the secondary current measurement is $<0.5 \mathrm{~A} / \mathrm{min}$ over the $7.8 \mathrm{~min}$ measurement time, which is negligible.

Note that the only way to detect drift in a closed loop system, i.e. during the measurement of a sample, is to observe whether the (non-zero) voltage over the sample is stable when the secondary current is at or around the critical current and is kept constant by the feedback system. The usually steep slope of the DC voltage-current transition of superconductors around the critical current results in a relatively high sensitivity of the sample voltage to current drift. However, the voltage-current slope in the samples tested so far was too low for a useful drift determination.

If the sample did not quench during the measurement, it is in principle possible to return the secondary current back to zero in a controlled way, but the primary current will then be larger then zero, as a result of resistive losses in the secondary that have occurred during the measurement. It is therefore not possible to determine the amount of drift in a closed loop system by observing the difference between the primary and secondary current when either of them is returned to zero, as is done above for an open loop system.

\section{Effective transfer function}

The theoretical gain $I_{\mathrm{s}} / I_{\mathrm{p}}$ is 1157 , as follows from (6). The measured initial ratio between the primary and secondary current is 877, as shown in Figure 10. The measured ratio is somewhat lower than the theoretical value of 1157 and reduces over time. The reason for this difference is twofold. First, the actual values for the self- and mutual inductances of the transformer coils and the load can differ from the calculated values. For example, the large sensitivity of the gain to the actual load inductance is clearly demonstrated in Figure 2. Secondly, the gain reduces as a result of the resistive losses that occur in the splices in the secondary. Even though the measured ratio between the secondary and primary current is lower

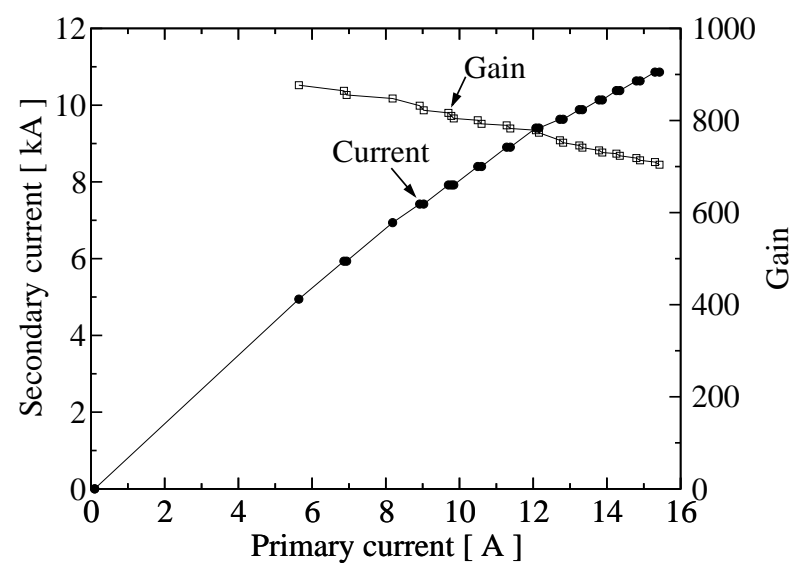

FIG. 10. Effective ratio between the primary and secondary current.

than the theoretical maximum gain, the obtained value is sufficient for all anticipated measurements.

\section{E. Cable voltage-current measurement}

To demonstrate the suitability of the superconducting transformer system as a current source for voltagecurrent transition measurements, data are presented on two bifilar $\mathrm{Nb}_{3} \mathrm{Sn}$ Rutherford cables, measured in series in an $11 \mathrm{~T}$ background magnetic field perpendicular to the broad side of the cables. The background magnetic field is provided by a $12 \mathrm{~T}$ split magnet at the NHMFL. The cables were manufactured at Lawrence Berkeley National Laboratory, Berkeley, CA, USA, (LBNL) for the US Large Hadron Collider Accelerator Research and Development Program (LARP). Similar cables, as well as results of comparable cable measurements, are more extensively described elsewhere ${ }^{9}$. The resulting cable voltagecurrent data, measured using a step-and-hold, i.e. DC method, are shown in Figure 11.

The expected critical current for these cables at $11 \mathrm{~T}$ is around $16 \mathrm{kA}$. It is seen that Cable 1 exhibits an early voltage rise, while Cable 2 is still entirely superconducting. The early voltage rise in Cable 1 is due to issues with the sample preparation. The sample voltages over Cable 1 show an initial voltage that contains an inductive component with a time constant of a few tens of seconds, after which the voltages stabilize to the static level as indicated in the graph.

Any drift in the secondary current depends, as in Section III C, on the accuracy of the offset determination in the 3 second period after resetting of the current meter system. Accurate offset determination can be disturbed by momentary noise that enters the integrator through pickup of electro-magnetic disturbances in the relatively noisy environment of a laboratory operating high field resistive magnets. It is therefore not possible to give a single value for the drift in the secondary. No sample 


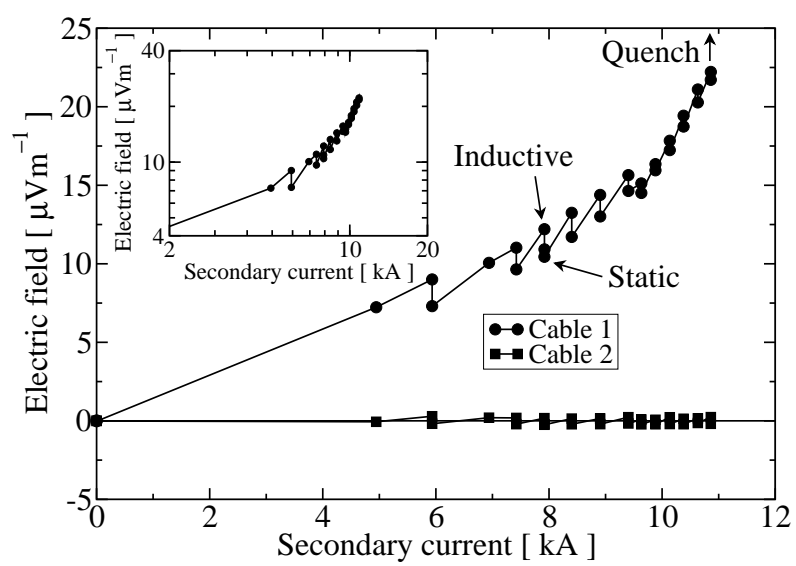

FIG. 11. Electric field as a function of transport current, measured on a $\mathrm{Nb}_{3} \mathrm{Sn}$ Rutherford cable at $11 \mathrm{~T}$ applied magnetic field. The inset shows the data for Cable 1 on a double logarithmic scale.

voltage drift, and therefore no current drift, was observable in the timeframe of the measurement of the static voltage in Figure 11, which was up to one minute. Based on the calibrations in Section IIIC it can be expected that the drift is negligible when the offset is accurately determined.

It is unfortunate that one of the cables in the sample showed an early transition to the normal state. Nonetheless, the resulting voltage-current transition demonstrates the successful commissioning of the superconducting transformer and control system as a reliable current source for such measurements.

\section{CONCLUSIONS}

A DC superconducting transformer system is designed, built, and successfully commissioned. The system uses a current measurement and feedback system that is constructed around a normal-conducting Rogowski coil and a low drift digital integrator. It is shown that the reproducibility in the current measurement system is better than $0.01 \%$ for currents up to $4 \mathrm{kA}$, and estimated to be better than $0.05 \%$ for currents up to $25 \mathrm{kA}$. The drift in the secondary current depends on accurate determination and compensation of the offset voltages in the digital integrator circuitry, but can be better than $0.5 \mathrm{~A} / \mathrm{min}$. An effective current gain of 877 is achieved, which reduces over time as a result of losses in the secondary and sample splices. The gain is lower than the design value and very sensitive to the inductance of the load, but is sufficiently high. The maximum current that was reached during system tests with a shorted secondary is $28 \mathrm{kA}$. This was limited by insufficient support of the secondary splice, and a desire to minimize the risk of accidental damage to the transformer, given the present absence of a need to test the transformer to its limit. Successful commissioning of the transformer as a high current source is demonstrated by a voltage-current measurement on a set of cables.

\section{ACKNOWLEDGMENTS}

The authors would like to thank D. A. Ward of Rocoil Ltd. for his help with the Rogowski coils, WangNMR Inc. for the fabrication of the primary coil, T. Stezelberger for his help with debugging of the FPGA code, X. Wang for his help with the Labview code, P. Pipersky for his help with the transformer mechanical design, and H. C. Higley and N. L. Liggins for their technical expertise and help with the transformer construction and test. This work was partly supported by the Director, Office of Science, High Energy Physics, US Department of Energy under contract no. DE-AC02-05CH11231.

${ }^{1}$ D. A. Ward and J. L. T. Exon, Eng. Sci. and Educ. J. June 1993, 105 (1993).

${ }^{2}$ H. H. J. ten Kate, W. Nederpelt, and L. J. M. van der Klundert, in Proc. $10^{\text {th }}$ Int. Cryog. Eng. Conf., Helsinki, Finland, edited by H. Collan, P. Berglund, and M. Krusius (Butterworth \& Co., Guildford, Surrey, UK, 1984) p. 712.

${ }^{3}$ H. H. J. ten Kate, W. Nederpelt, P. Juffermans, F. van Overbeeke, and L. J. M. van der Klundert, Adv. Cryo. Eng. 31, 1309 (1986).

${ }^{4}$ H. H. J. ten Kate, H. W. Weijers, and J. M. van Oort, IEEE Trans. Appl. Supercond. 3, 1334 (1993).

${ }^{5}$ H. W. Weijers, A. Godeke, B. ten Haken, S. Wessel, and H. H. J. ten Kate, Adv. Cryo. Eng. 39, 1147 (1994).

${ }^{6}$ H. G. Knoopers, W. A. J. Wessel, H. J. G. Krooshoop, O. A. Shevchenko, A. Godeke, H. H. J. ten Kate, B. A. Smith, R. J. Camille, and J. Minervini, in Proc. 16th Int. Cryog. Eng. Conf. (ICEC), Kitakyushu, Japan (1997) p. 803.

${ }^{7}$ A. P. Verweij, C.-H. Denarie, S. Geminian, and O. Vincent-Viry, Inst. of Physics Conference Series 43, 833 (2006).

${ }^{8}$ B. Ni, S. Hakoda, E. S. Otabe, and M. Kiuchi, Inst. of Physics Conference Series 43, 837 (2006).

${ }^{9}$ D. R. Dietderich and A. Godeke, Cryogenics 48, 331 (2008). 